\title{
918 DOXYCYCLINE REGULATABLE B-CATENIN DEMONSTRATES INDUCIBLE IMMUNE EVASION IN A MELANOMA GEM MODEL
}

${ }^{1}$ Alexandra Cabanov, ${ }^{2}$ Stefani Spranger, ${ }^{1}$ Thomas Gajewski, ${ }^{1}$ Alexandra Cabanov*, ${ }^{3}$ Elen Torres-Mejia. 'University of Chicago, Chicago, USA; ${ }^{2}$ Koch Institute, Massachusetts Institute of Technology, Ragon Institute, Cambridge, MA, USA; ${ }^{3}$ Koch Institute, Ragon Institute, Cambridge, MA, USA

Background Lack of response to checkpoint blockade immunotherapy has been linked to a deficiency of immune cell infiltration within the tumor microenvironment (TME). One demonstrated mechanism sufficient for the non-T cell inflamed TME is tumor cell-intrinsic activation of the $\beta$-catenin signaling pathway. Using genetically engineered mouse models (GEMMs), tumors constitutively expressing active $\beta$-catenin lack a robust endogenous $T$ cell infiltrate and fail to respond to immunotherapies. In support of these mouse studies, human melanoma metastases with increased active $\beta$-catenin signaling exhibit decreased numbers of tumor infiltrating Batf3-driven cDC1 and CD3 + T cells. However, whether temporal activation and inactivation of $\beta$-catenin within the same developing tumor would alter immune cell infiltration is not known.

Methods A model was created in which tamoxifen-regulated Cre-recombinase mediates BRAFV600E oncogene activation and PTEN tumor suppressor gene deletion as well as expression of a doxycycline regulatable reverse transactivator. Upon administration of doxycycline via the drinking water to these animals, a non-degradable form of nuclear $\beta$-catenin becomes expressed. Immunofluorescence assays were performed assessing the $\beta$-catenin expression status in the tumor cells as well as immune cell infiltration within the TME. Additionally, immunotherapy efficacy experiments were performed.

Results We observed that administration of doxycycline to these animals drove expression of an active form of nuclear $\beta$-catenin. Activation of nuclear $\beta$-catenin resulted in a 2 -fold decrease in the overall $\mathrm{CD} 3+\mathrm{T}$ cells infiltration into the TME. Moreover, this decrease in immune infiltration also resulted in loss of anti-PD-L1 + anti-CTLA-4 therapy efficacy. We next performed studies assessing the kinetics with which $\beta$-catenin levels diminish upon doxycycline removal. Switching animals to regular drinking water resulted in rapid reduction of nuclear $\beta$-catenin levels, including 50 percent reduction after two days of doxycycline removal and almost complete reduction of nuclear $\beta$-catenin after four days.

Conclusions We describe a novel mouse model in which we induce autochthonous melanoma tumors in mice along with inducible expression of a non-degradable, nuclear $\beta$-catenin modulated by doxycycline in the drinking water. Activation of $\beta$-catenin signaling in melanoma tumors resulted in reduction of immune cells in the TME as well as loss of checkpoint blockade immunotherapy efficacy. This activation can be rapidly reversed by removing doxycycline, allowing for future studies evaluating the consequences of turning off $\beta$-catenin once it has already driven a non-T cell-inflamed TME.

Acknowledgements This work was supported by the Wissler Fellowship from the University of Chicago (SS) K99/R00 (NCI; SS), and R35CA210098 (TG).

http://dx.doi.org/10.1136/jitc-2021-SITC2021.918 